\title{
A Simple Chaotic Map Model for Fractal Traffic Generation in High-speed Computer Networks
}

\author{
G. Millán \\ Facultad de Ingeniería y Tecnología \\ Universidad San Sebastián \\ Puerto Montt, Chile \\ ginno.millan@uss.cl
}

\begin{abstract}
An extension of the models used to generate fractal traffic flows is presented by means of the formulation of a model that considers the use of one-dimensional chaotic maps. Based on the disaggregation of the temporal series generated by the model, a valid explanation of behavior of the values of Hurst exponent is proposed and the feasibility of their control from the parameters of the proposed model is shown.
\end{abstract}

Keywords-Chaotic maps; Fractality; Hurst exponent (H); Selfsimilarity; Traffic modeling in high-speed computer networks.

\section{INTRODUCTION}

The chaotic behavior of systems is an intermediate paradigm between two dogmatic scientific and philosophical concepts of the universe: absolute knowledge upheld by determinism, and total ignorance at the hands of randomness. Paradoxically, and supported by the existence of the above two dogmatic positions, an assertion as natural as the one made points at the main failure in the analysis of systemic behaviors: the extended use of dichotomies to characterize them.

It is in this scenario that the theory of chaos, defined by Kellert as the qualitative study of periodic and unstable behavior in deterministic and non-linear dynamic systems [1], invades and establishes the omnipresence of unpredictability as a fundamental trait of common experience [2]. Then, the theory of chaos, instead of trying to understand the behavior of systems in a merely quantitative manner to determine exactly their future states, it concerns with understanding of a long-term behavior, searching for patterns under a holistic philosophy rather than a reductive philosophy.

As can be seen and inferred from the ideas given above, and in full agreement with the spirit of this research, it is neither possible nor practical to approach the problem of the characterization of the behavior of the systems of interest considering the full conceptual extension of the theory of chaos, and for that reason it is accepted that chaos is the phenomenon by which low-order non-linear systems show an apparently random complexity and behavior [3]. These systems are of low-order because they can be described correctly by a reduced number of variables and parameters. They are also dynamic systems, i.e., with the variables of interest, which are deterministic, evolve over time, because the values of those variables at any instant of time can be determined only from their previous values given a set of dynamic laws. Finally, those dynamic laws that describe the system evolution in time are non-linear [4].

At this point it is convenient to make it clear that chaotic systems differ from conventional dynamic systems in the sense that they are intrinsically unpredictable, a fact that is evident even when its subjacent dynamic laws are of a deterministic character. But the above does not have to lead to the belief that chaos implies unpredictability, since that is only partially true because of existence of two main sources of unpredictability, namely the inaccuracy of the initial data, and its origin as a characteristic inherent to certain nonlinear relations between numerical variables [5]. Therefore, the definition of chaos as a property of a system refers to its sensitivity to the initial conditions, i.e. that given two trajectories arbitrarily close to one another in the phase space of a chaotic system, they diverge at an exponential rate given by the Lyapunov global exponent.

Note that it is certainly paradoxical for an essentially deterministic system, with deterministic dynamic laws, to show a chaotic behavior, since the basic premise of dynamic systems is that the knowledge of the initial conditions makes possible the determination of the system future behavior at any time. In practice, the initial conditions can only be specified with finite precision. These uncertainties introduced in the initial conditions for case of chaotic systems increase exponentially, and that explains the unpredictability of their behavior. Strictly, chaos involves the possibility of making good short-term predictions, but it makes impossible any long-term prediction of a practical order [6]. A direct result of the above is that very simple systems, even with only one degree of freedom, can give rise to surprisingly complex behaviors.

The notion of chaos often appears linked to the notion of fractal introduced by Mandelbrot [7], and even though it has not been proved rigorously, fractal properties seem inherent in chaotic processes, so apparently chaos and fractal sets are independent and unrelated concepts [8]. However, keeping in mind that the fractal dimension concept raises a generalization of the notion of dimension through the introduction of the noninteger values for their specification, an extensively reported fact in its applications [9]; unexpectedly all chaotic systems tend to evolve asymptotically in their phase space toward a bounded region called strange attractor that has a non-integer dimension, i.e., a fractal. It can thus be argued that very often 
the strange attractors are fractals in their nature and are capable of exhibiting their complexity over different time or space scales. Because of the above it is therefore possible to state that the concepts of fractal geometry can be used to describe the evolutionary characteristics of chaotic systems, and chaotic systems in turn can be used conveniently as generators of fractal structures, thereby implying self-similarity and therefore its characterization index: Hurst exponent $(H)$.

It should be pointed out that since there is no simple definition of fractals, they are generally defined in terms of their attributes, such as, for example, the slow decay of their variances, the hyperbolic tail distribution of the time density between successive arrivals, the infinite order moments or poorly defined statistics, $1 / f$ noise, long-range dependence, selfsimilarity, and the previously mentioned non-integer dimension, among others [10], [11]. The presence of such characteristics in the traffic flows of actual high-speed computer networks is, therefore, the ultimate aim of the whole discussion presented.

The statistically self-similar behavior of traffic flows in the present high-speed computer networks is a fact that has been extensively reported for different levels of telematic systems coverage, transmission technologies, wireless systems, control and signaling protocols, ATM queueing network behavior, and applications, particularly in video [12].

Similarly, the problem of the characterization of traffic has received considerable attention in the literature, giving rise to a number of proposals of stochastic models, see [13]-[18].

However, and in spite of all the efforts underlying the arguments and methodologies stated above, two problem situations inherent in the generation of traffic with long-range dependence are ubiquitous, namely the degree of representatives of Hurst exponent as a unique parameter for characterizing its effects on the performance of the tails systems in which it appears, and the behavior shown by its value in the self-similar second order series obtained within the interval of interest for $H ; 0.5<H<1$. In this respect, [19] shows in an isolated way both problems and their implications.

\section{CHAOTIC MAPS AND SELF-SIMILAR TRAFFIC}

The use of chaotic maps as models of traffic was proposed for the first time in [3], starting from the pioneering work [20], and in essence a chaotic map is a variant of the On/Off traffic model of [21], with the basic difference that its basis lies in the discrete dynamics instead of in the probabilistic approaches.

A chaotic system characterized by a certain non-linear function $f: D \rightarrow D$ with $D \in \mathbb{R}^{m}$ is available. The system evolves according to the process equation, defined by $f$, which for a discrete system is given by

$$
\mathbf{x}[k]=f(\mathbf{x}[k-1], u[k-1] ; \boldsymbol{\theta})+\mathbf{v}[k],
$$

where, $\mathbf{x}[k]$ is the system state vector, $u[k]$ the input excitation, $\mathbf{v}[k]$ the noise vector of the process, and $\boldsymbol{\theta}$ is the vector of the system parameters.

In general, the state of the system cannot be observed directly, so samples of it (or of functions of it) are required that are obtained by means of a measuring process given by

$$
\mathbf{y}[k]=g(\mathbf{x}[k])+\mathbf{w}[k], \text { with } k=0, \ldots, N,
$$

where $g$ is the measurement function (which can be known or not), $\mathbf{y}[k]$ is the measurements vector, and $\mathbf{w}[k]$ is the noise vector of the measurement.

A chaotic map is an application $f: X \rightarrow Y$ that associates each element $x \in X \subseteq \mathbb{R}^{m}$ with a single element $y \in Y \subseteq \mathbb{R}^{q}$, where $X$ is the domain of $f$ and $Y$ is the arrival set of $f$.

Let $f: D \rightarrow D$ be an application with $D \in \mathbb{R}^{m}$. An iterated map is the system formed by the set of $m$ equations given by

$$
\mathbf{x}[n]=f(\mathbf{x}[n-1] ; \boldsymbol{\theta}) .
$$

As seen from (3), a chaotic map is a class of discrete autonomous system, and this work deals exclusively with onedimensional maps, a fact for which it is convenient to establish that a one-dimensional chaotic map is an application $f: D \rightarrow D$, with $D \subseteq \mathbb{R}$ such that

$$
x[n]=f(x[n-1] ; \theta) .
$$

The definition of chaos as a property of a chaotic system refers to its sensitivity to the initial conditions. Considering a chaotic map defined by $x_{n+1}=f(x)$ and two trajectories with almost identical initial conditions $x_{0}$ and $x_{0+\varepsilon}$, where $\varepsilon \rightarrow 0$, its sensitivity to the initial conditions is described by

$$
\left|f^{N}\left(x_{0}+\varepsilon\right)-f^{N}\left(x_{0}\right)\right|=\varepsilon \exp \left(N \lambda\left(x_{0}\right)\right),
$$

where $f^{N}(\cdot)$ represents the $N^{\text {th }}$ iteration of the map, and $\lambda\left(x_{0}\right)$ is Lyapunov global exponent which describes the exponential divergence. For the map to be chaotic this parameter must be positive for most of the $x_{0}$ [22]. Then (5) implies which points that begin with similar initial conditions develop along different trajectories.

Let $f: I \rightarrow I$ be an application. It is said that $f(x)$ is a onedimensional piecewise affine map (PWA) if there is a finite number of points $e_{0}<\ldots<e_{M}$, such that the interval $I=\left[e_{0}, e_{M}\right]$ can be subdivided into $M$ smaller intervals $E_{i}=\left[e_{i-1}, e_{i}\right)$, with $i=1, \ldots, M-1$ and $E_{M}=\left[e_{M-1}, e_{M}\right]$, within which $f(x)$ is affine. Mathematically, a PWA map is expressed as

$$
f(x)=\sum_{i=1}^{M}\left(a_{i} x+b\right) \Psi_{E_{i}}(x),
$$

where $\Psi$ denotes the characteristic function defined as follows.

Let $\Psi_{R}: D \rightarrow\{0,1\}$ be an application with $D \subseteq \mathbb{R}$. It is said that $\Psi_{R}$ is the characteristic function of region $R$ if

$$
\Psi_{R}=\left\{\begin{array}{ll}
1, & x \in R \\
0, & x \notin R
\end{array} .\right.
$$

In other words, $f(x)$ has a similar behavior within each of the $M$ intervals $E_{i}$ into which the arrival interval $I$ is divided as if it were observed in the whole interval $I$.

A one-dimensional map composed of two intervals, in which the state variable $x_{n}$ evolves in time according to two functions $f_{1}(\cdot)$ and $f_{2}(\cdot)$ that satisfy condition (5), allows writing it, from the development of (4), as 


$$
x_{n+1}=\left\{\begin{array}{ll}
f_{1}\left(x_{n}\right), & 0<x_{n} \leq d \\
f_{2}\left(x_{n}\right), & d<x_{n}<1
\end{array} .\right.
$$

This notation makes it possible to conceive a frame generation process considering that the source of origin is in a passive state or in an active state (in a similar way as the case of the On/Off models) at a given moment $n$, as a function of whether the value of the state variable $x_{n}$ is above or below a certain activation threshold $d$. In this way all the iterations of the map in the active state correspond to frame (burst of frames) generation processes, and all the iterations of the map in the passive state to time processes between successive arrivals.

Under the same reasoning, and considering the permanence of the map in one or the other previous states, the evolution of the mesh arrival process is described from the characteristic function (7), which, for the sake of consistency with the notation used in (8), can be written as

$$
y_{n}=y\left(x_{n}\right)=\left\{\begin{array}{ll}
0, & 0<x_{n} \leq d \\
1, & d<x_{n}<1
\end{array} .\right.
$$

An interesting interpretation of the above model is reported in [23] considering it formed by two dynamic layers, one hidden given by $x_{n}$ and one visible specified by $y_{n}$.

It is particularly interesting to see that the behavior of a trajectory over which the map given by (8) evolves is such that it does not have to visit the two regions of its phase space with equal frequency, and furthermore, there is no reason to consider, even within the same attractor, a uniform probability density function of the generated sequences, so it is reasonable to ask about the frequency with which a given trajectory visits each region of the map in an observation interval of $n$ iterations, as well as about the way of calculating such probability density function from an initial condition $x_{0}$.

In this respect, the reply to both questions is found in the density distribution of the map states, $\rho_{n}(x)$, [24]

$$
\rho_{n}=\frac{1}{N} \sum_{i=1}^{N} \delta\left[x-x_{n}(i)\right],
$$

where $\delta(x)$ is Dirac delta function and the evolution of $\rho_{n}(x)$ is in agreement with the equation of Frobenius-Perron [25]

$$
\rho_{n+1}(x)=\int \delta[x-f(z)] \rho_{n}(z) d z .
$$

In the case of a one-dimensional map given by $x_{n+1}=f\left(x_{n}\right)$, with $x_{n} \in[0,1], n=0,1,2, \ldots$, from (10) we have that

$$
\rho(x)=\lim _{N \rightarrow \infty} \frac{1}{N} \sum_{n=0}^{N} \delta\left[x-f_{n}\left(x_{0}\right)\right] .
$$

If $\rho(x)$ does not depend on $x_{0}$, the system is ergodic [24], and therefore it is true that

$$
\lim _{N \rightarrow \infty} \frac{1}{N} \sum_{i=0}^{N} g\left(x_{i}\right) \equiv \lim _{N \rightarrow \infty} \frac{1}{N} \sum_{i=0}^{N} g\left[f^{i}\left(x_{0}\right)\right]=\int_{0}^{1} \rho(x) g(x) d x .
$$

However, since $\rho_{n}(x)$ must be stationary because of (13), i.e., it does not have to depend on instant $n$, it is known as the invariant density of map $f(x)$ [26], and it describes the iteration density of $x_{n}$ in the interval $(0,1)$ when $n \rightarrow \infty$. Therefore, $\rho_{n}(x)$ is a self-function of the Frobenius-Perron operator with selfvalue 1 , and therefore

$$
\rho(x)=\int_{0}^{1} \delta[x-f(z)] \rho(z) d z .
$$

A concise presentation of the set of facts, that give rise to the treatment of traffic considering its self-similar and LRD nature, converge in the need to capture its fluctuations on different time scales in order to make assertive forecasts of the yield of communications systems. In this respect, the selfsimilarity contributes with the parsimony required to specify the statistical details of the variables involved from a minimum set of modeling parameters, and a model based on the theory of complexity contributes the robustness needed to cohere those parameters with information.

\section{SPeCification Of THE ChaOtic Model}

Consider the nonlinear double intermittence map

$$
x_{n+1}=\left\{\begin{array}{cc}
\varepsilon_{1}+x_{n}+c_{1} x_{n}^{m_{1}} & , 0<x_{n} \leq d \\
-\varepsilon_{2}+x_{n}-c_{2}\left(1-x_{n}\right)^{m_{2}}, & d<x_{n}<1
\end{array},\right.
$$

where $c_{1}=\left(1-\varepsilon_{1}-d\right) / d^{m_{1}}, c_{2}=\left(d-\varepsilon_{2}\right) /(1-d)^{m_{2}}$ [4].

If $m_{2}=1$ and $\varepsilon_{2}=0$, from (15) we get the model of a nonlinear chaotic intermittent map [3]

$$
x_{n+1}=\left\{\begin{array}{ll}
\varepsilon+x_{n}+c x_{n}^{m} & , 0<x_{n} \leq d \\
\left(x_{n}-d\right) /(1-d), & d<x_{n}<1
\end{array},\right.
$$

where $c=(1-\varepsilon-d) / d^{m}$, with $\varepsilon \square d$.

The map parameters $(\varepsilon, m$, and $d$ ) are used to control the probability of permanence in the inactive state, the traffic load; i.e., the mean rate of mesh arrival, and the degree of selfsimilarity density, $H$. Specifically, the fit of the $\varepsilon$ parameter incides on the probability of permanence of the iterations of the map in the inactive state, while the traffic load depends on parameters $m$ and $d$ [27].

The condition $\varepsilon \square d$ is well defined, at least theoretically, if $\varepsilon=0$, which leads to the control of the limit of the range of temporal scales over which LRD is observed. In [27] it is shown that if $\varepsilon=0$, permanence time can be of any length, but as $\varepsilon$ increases above 0 , the escape time from the region tends to a fixed upper limit.

Considering $m=1$ with the purpose of decreasing the degree of the function that characterizes the inactive state of the map implies generating traffic with short-range dependence (SRD), which is verified from the relation existing between $H$ and $m$ given by $H=(3 m-4) /(2 m-2)$, with $m=\max \left\{m_{1}, m_{2}\right\}$ [28], i.e., $H=0.5$. In other words, a geometric type decay takes place for the map regions, which is synonymous of uncorrelated traffic, i.e., traffic with SRD.

Therefore, the proposed map together with its characteristic function is given by 


$$
x_{n+1}=\left\{\begin{array}{ll}
x_{n}+x_{n}^{m} & , 0<x_{n} \leq d \\
\left(x_{n}-d\right) / d^{m}, & d<x_{n}<1
\end{array},\right.
$$

and

$$
y_{n}=y\left(x_{n}\right)=\left\{\begin{array}{ll}
0, & 0<x_{n} \leq d \\
1, & d<x_{n}<1
\end{array} .\right.
$$

Rewriting (17) in the form

$$
x_{n+1}=\left\{\begin{array}{ll}
f_{1}(x)=x_{n}+x_{n}^{m} & , 0<x_{n} \leq d \\
f_{2}(x)=\left(x_{n}-d\right) / d^{m}, & d<x_{n}<1
\end{array},\right.
$$

from the development of (14) applying (19) we get

$$
\rho(x)=\int_{0}^{d} \delta\left[x-f_{1}(x)\right] \rho(z) d z+\int_{d}^{1} \delta\left[x-f_{2}(x)\right] \rho(z) d z,
$$

an equation for which Schuster [22] establishes the existence of only one physically relevant solution that is obtained from taking $z=1 / f_{i}(x)$, i.e.,

$$
\rho(x)=\frac{\rho\left(f_{1}^{-1}(x)\right)}{f_{1}^{\prime}\left(f_{1}^{-1}(x)\right)}+\frac{\rho\left(f_{2}^{-1}(x)\right)}{f_{2}^{\prime}\left(f_{2}^{-1}(x)\right)},
$$

where $f_{1}^{\prime}$ represents the first derivate of $f_{1}$. Then the expression for the invariant density is given by

$$
\rho(x)=\sum_{i=1}^{2} \frac{\rho\left(y_{i}\right)}{f_{i}^{\prime}\left(y_{i}\right)}, \text { with } y_{i}=f_{i}^{\prime}(x),
$$

which allows obtaining the system load, i.e., the probability of remaining in active state, from the integration of (22) between the limits $d$ and 1 .

\section{EXPERIMENTAL RESULTS}

The self-similar behavior with LRD of the traffic generated by the proposed map is confirmed by means of the calculation of Hurst exponent using the rescaled range analysis $(\mathrm{R} / \mathrm{S})$ and the aggregate variance analysis (Var) [29].

In the $\mathrm{R} / \mathrm{S}$ analysis the value of $H$ is obtained directly from the slope of the logarithmic plot, while in the Var analysis $H$ is obtained from the relation $H=1-\beta / 2$ where $0<\beta<1$ [30].

In this first stage of research no online traffic is generated because we are dealing only with the validation of model; and instead of it all algorithms are programmed in MATLAB.

As a first point, two values are specified in Table I for parameter $m$, keeping the number of the map iterations $N$ and parameter $d$ fixed.

Tables II to IV show experiments varying parameters $m$ and $d$ for a constant number of iterations of the model, to obtain the Hurst parameter $H$.

Fig. 1 and Fig. 2 show the behavior of $x_{n+1}$ and $y_{n}$ for the data of Table I. Fig. 1(b) shows only the first 80 iterations for $y_{n}$, since the rest, up to iteration $N=1000$, are equal to zero.

\begin{tabular}{|c|c|c|c|c|}
\hline \multirow{2}{*}{$N$} & \multirow{2}{*}{$d$} & \multirow{2}{*}{$m$} & \multicolumn{2}{|c|}{$\boldsymbol{H}$} \\
\hline & & & $\mathrm{R} / \mathrm{S}$ & Var \\
\hline \multirow{6}{*}{1000} & \multirow{6}{*}{0.5} & 1 & 0.50 & 0.42 \\
\hline & & 1.2 & 0.51 & 0.46 \\
\hline & & 1.4 & 0.54 & 0.50 \\
\hline & & 1.6 & 0.68 & 0.58 \\
\hline & & 1.8 & 0.73 & 0.68 \\
\hline & & 2 & 0.87 & 0.78 \\
\hline
\end{tabular}

Fig. 3 shows the graphic aspect of the traffic generated for $N=1000$ iterations, $d=0.5$ and $m=2$ (for the case of Fig. 2).

\begin{tabular}{|c|c|c|c|c|}
\hline \multirow{2}{*}{$N$} & \multirow{2}{*}{$d$} & \multirow{2}{*}{$m$} & \multicolumn{2}{|c|}{$\boldsymbol{H}$} \\
\hline & & & $\mathrm{R} / \mathrm{S}$ & Var \\
\hline \multirow{6}{*}{1000} & \multirow{6}{*}{0.3} & 1 & 0.50 & 0.44 \\
\hline & & 1.2 & 0.51 & 0.47 \\
\hline & & 1.4 & 0.61 & 0.56 \\
\hline & & 1.6 & 0.72 & 0.65 \\
\hline & & 1.8 & 0.78 & 0.72 \\
\hline & & 2 & 0.90 & 0.82 \\
\hline
\end{tabular}

TABLE I. SPECIFICATION OF THE MOdEL PARAMETERS

\begin{tabular}{|c|c|c|c|}
\hline $\mathrm{N}$ & $\mathrm{d}$ & $\mathrm{m}$ & Show in \\
\hline \multirow{2}{*}{1000} & \multirow{2}{*}{0.5} & 1 & Fig. 1 \\
\cline { 3 - 4 } & & 2 & Fig. 2 \\
\hline
\end{tabular}

\begin{tabular}{|c|c|c|c|c|}
\hline \multirow{2}{*}{$N$} & \multirow{2}{*}{$d$} & \multirow{2}{*}{$m$} & \multicolumn{2}{|c|}{$\boldsymbol{H}$} \\
\hline & & & $\mathbf{R} / \mathbf{S}$ & Var \\
\hline \multirow{6}{*}{1000} & \multirow{6}{*}{0.1} & 1 & 0.51 & 0.44 \\
\hline & & 1.2 & 0.53 & 0.50 \\
\hline & & 1.4 & 0.66 & 0.56 \\
\hline & & 1.6 & 0.75 & 0.65 \\
\hline & & 1.8 & 0.81 & 0.72 \\
\hline & & 2 & 0.92 & 0.82 \\
\hline
\end{tabular}

TABLE II. SPECIFICATION OF THE MODEL PARAMETERS

TABLE III. SPECIFICATION OF THE MODEL PARAMETERS

TABLE IV. SPECIFICATION OF THE MODEL PARAMETERS
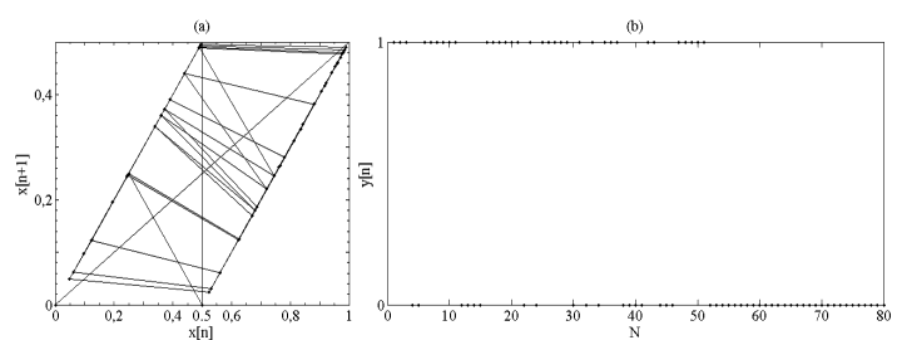

Figure 1. Behavior of the model considering $N=1000, d=0.5$, and $m=1$.
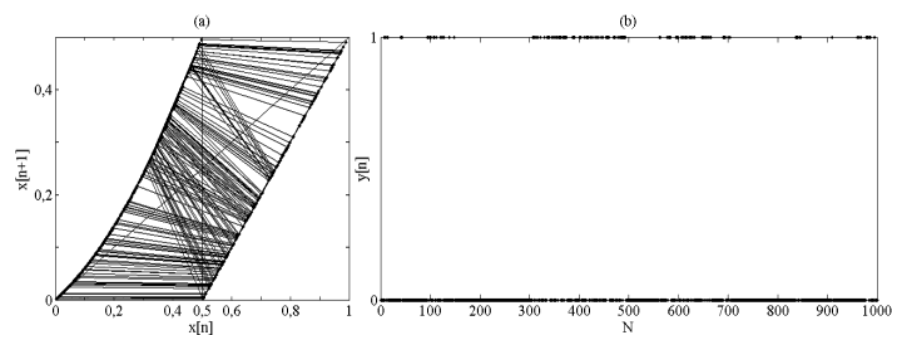

Figure 2. Behavior of the model considering $N=1000, d=0.5$, and $m=2$. 


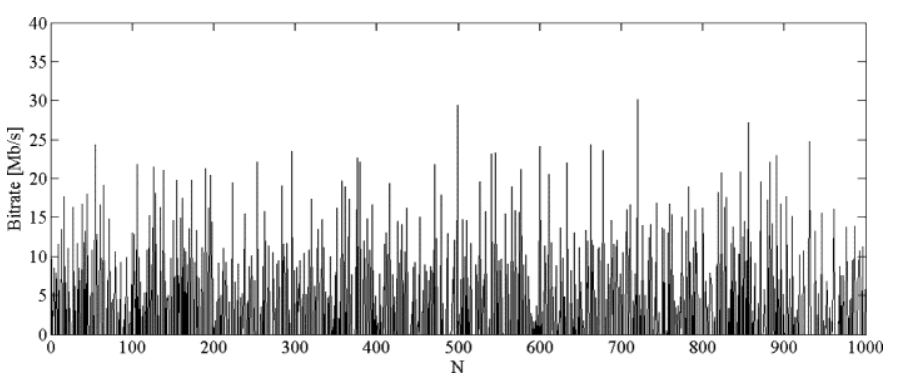

Figure 3. Traffic generating by the map. $N=1000, d=0.5$, and $m=2$.

Tables V to VII present values of $H$ for random traffic sequences extracted from the original series used to formulate Tables II to IV. These random sequences are formed from the extraction of the number of samples specified under Random $N$, and they are then analyzed as samples of traffic generated directly by the model.

TABLE V. HURST EXPONENT FOR DISSAGREGATED SERIES

\begin{tabular}{|c|c|c|c|c|c|}
\hline \multirow{2}{*}{$N$} & \multirow{2}{*}{ Rand. $N$} & \multirow{2}{*}{$d$} & \multirow{2}{*}{$m$} & \multicolumn{2}{|c|}{$\boldsymbol{H}$} \\
\hline & & & & $\mathrm{R} / \mathrm{S}$ & Var \\
\hline \multirow{6}{*}{1000} & \multirow{6}{*}{500} & \multirow{6}{*}{0.5} & 1 & 0.42 & 0.37 \\
\hline & & & 1.2 & 0.45 & 0.43 \\
\hline & & & 1.4 & 0.51 & 0.48 \\
\hline & & & 1.6 & 0.55 & 0.52 \\
\hline & & & 1.8 & 0.65 & 0.58 \\
\hline & & & 2 & 0.72 & 0.66 \\
\hline
\end{tabular}

TABLE VI. HURST EXPONENT FOR DISSAGREGATED SERIES

\begin{tabular}{|c|c|c|c|c|c|}
\hline \multirow{2}{*}{$N$} & \multirow{2}{*}{ Rand. $N$} & \multirow{2}{*}{$d$} & \multirow{2}{*}{$m$} & \multicolumn{2}{|c|}{$\boldsymbol{H}$} \\
\hline & & & & $\mathbf{R} / \mathbf{S}$ & Var \\
\hline \multirow{6}{*}{1000} & \multirow{6}{*}{500} & \multirow{6}{*}{0.3} & 1 & 0.44 & 0.38 \\
\hline & & & 1.2 & 0.47 & 0.45 \\
\hline & & & 1.4 & 0.53 & 0.51 \\
\hline & & & 1.6 & 0.62 & 0.57 \\
\hline & & & 1.8 & 0.67 & 0.62 \\
\hline & & & 2 & 0.75 & 0.67 \\
\hline
\end{tabular}

TABLE VII. HURST EXPONENT FOR DISSAGREGATED SERIES

\begin{tabular}{|c|c|c|c|c|c|}
\hline \multirow{2}{*}{$N$} & \multirow{2}{*}{ Rand. $N$} & \multirow{2}{*}{$d$} & \multirow{2}{*}{$m$} & \multicolumn{2}{|c|}{$\boldsymbol{H}$} \\
\hline & & & & $\mathrm{R} / \mathrm{S}$ & Var \\
\hline \multirow{6}{*}{1000} & \multirow{6}{*}{500} & \multirow{6}{*}{0.1} & 1 & 0.43 & 0.38 \\
\hline & & & 1.2 & 0.45 & 0.42 \\
\hline & & & 1.4 & 0.52 & 0.50 \\
\hline & & & 1.6 & 0.57 & 0.52 \\
\hline & & & 1.8 & 0.63 & 0.57 \\
\hline & & & 2 & 0.71 & 0.66 \\
\hline
\end{tabular}

\section{DISCUSSION OF RESULTS}

The model shows that the generation of self-similar traffic with LRD can be approached from the control of parameters $m$ and $d$ considering a fixed number of iterations, as shown from the results given in Tables II to IV. However, because of its simplified present formulation, control of the permanence times in each state is not possible. In that respect, a general model like that of [26] does allow that with good accuracy. A simple example to illustrate that need is built on the basis of requiring that the traffic of a system should have a certain $H$, for example 0.90. Examining Table III and Table IV it is found that this requirement can only be satisfied by means of a change in the boundary of the iteration regions, which is impractical because of its repercussions on the characteristic function. It is a need that requires urgent attention.

Control of the degree of self-similarity of the generated traffic seems to be effective for the subjacent temporal series. The analysis of the results shown in the Tables V to VII is fundamental because it reflects the underlying problem: controlling the effect of locality on Hurst exponent. It is seen that the traces formed from random sections of the original samples reflect the behavior of the whole, and also that Hurst exponent can be considered by itself as a valid indicator to characterize the effects of the generated self-similar traffic with LRD on the performance of the tail systems in which it appears. The lack of a formal mathematical proof of this fact is acknowledged, but in a first instance, based on the effect that the characteristic function has on the perception of the system, an appropriate substantiation is found.

\section{CONCLUSIONS}

The feasibility of having an efficient and effective selfsimilar traffic generator from the parsimony of its model is shown, a fact made evident by an adequate control of the value of Hurst exponent. Furthermore, Hurst exponent is shown to be completely valid as a representative parameter to characterize self-similar traffic.

It is shown that the value of Hurst exponent in the disaggregation of self-similar temporal series depends on the original model of the traffic. In this respect, based on subsets of samples obtained randomly, it is seen that its value shows a tendency to remain constant and in that way adequately characterize the segments in question.

Finally, the most relevant fact for the future development of this research is the practical proof that $m=1$ must be discarded as an option for the formulation of a chaotic model generator of self-similar traffic because it inevitably leads to SRD traffic, as shown by the results given in Tables II to VII, so it is shown to be impossible to work only with a linear model.

\section{ACKNOWLEDGMENT}

Special thanks to the Advanced Human Capital Training Program of CONICYT 2012 for the support given to the author G. Millán through doctoral fellowship to study in Chile, and also to the VRID USACH for their support to this research.

\section{REFERENCES}

[1] S. H. Kellert, In the Wake of Chaos. Chicago, IL: The Univ. Chicago Press, 1993, p.2.

[2] S. Male, "Finding a horseshoe on the beaches of Rio," The Mathematical Intelligencer, vol. 20, no. 1, pp. 39-44, Mar. 1998.

[3] A. Erramilli, R. P. Singh, and P. Pruthi, "Chaotic maps as models of packet traffic," in The Fundamental Role of Teletraffic in the Evolution of Telecommunications Networks, J. Labetoulle and J. W. Roberts, Eds. Amsterdam, Netherlands: Elsevier, 1994, pp. 329-338.

[4] A. Erramilli, R. P. Singh, and P. Pruthi, "Modeling packet traffic with chaotic maps," Royal Inst. Tech., Stockholm, Sweden, Tech. Rep. ISRN KTH/IT/R-94/18-SE, Aug. 1994.

[5] M. Mahner, and M. Bunge, Fundamentos de Biofilosofía, México: Ed. Siglo XXI, 2000, pp. 220-221. 
[6] D. Luengo, "Estimación óptima de secuencias caóticas con aplicación en comunicaciones," Ph.D. dissertation. Dept. Ing. Comunicaciones. Univ. Cantabria, España, 2006.

[7] B. B. Mandelbrot, The Fractal Geometry of Nature, New York: W. H. Freeman and Company, 1983.

[8] C. J. Quintero. (2005, Feb.). Visualización de Atractores Extraños de Sistemas Dinámicos Caóticos. Univ. Málaga, Málaga, España [Online]. Available: http://www.lcc.uma.es.

[9] J. Lévy-Véhel and E. Lutton, Eds., Fractals in Engineering. New Trends in Theory and Applications. Germany: Springer, 2005.

[10] P. Park and W. Willinger, Eds.,Self-similar Network Traffic and Performance Evaluation. New York: Wiley, 2000.

[11] O. I. Sheluhin, S. M. Salmoslky, and A. V. Osin, Self-Similar Processes in Telecommunications, Chichester, England: Wiley, 2007.

[12] R. Narashima and R. Rao, "Modeling variable bit rate video on wired and wireless network using discrete-time self-similar systems," in 2002 IEEE Int. Conf. Personal Wireless Commun., pp. 290-294.

[13] D. P. Heyman and D. Lucantoni, "Modeling multiple IP traffic streams with rate limits," IEEE/ACM Trans. Netw., vol. 11, no. 6, pp. 948-958, Dec. 2003.

[14] S. Shah-Heydari and T. Le-Ngoc, "Parameter estimation of switched Poisson process models for short- and long-range dependent traffic," in IEEE Canadian Conf. Elect. Comput. Eng., 2003, vol. 2, pp. 1035-1038.

[15] B. K. Ryu and S. B. Lowen, "Point process approaches to the modeling and analysis of self-similar traffic. I. Model construction," in Proc. IEEE INFOCOM '96, San Francisco, CA, 1996, vol. 3, pp. 1468-1475.

[16] X. Yang and A. P. Petropulu, "The extended alternating fractal renewal process for modeling traffic in high-speed communication networks," IEEE Trans. Signal Process., vol. 49, no. 7, pp. 1349-1363, Jul. 2001.

[17] J. Yu, A. A. Petropulu, and H. Sethu, "Rate-limited EAFRP-a new improved model for high-speed network traffic," IEEE Trans. Signal Process., vol. 53, no. 2, pp. 505-522, Feb. 2005.

[18] D. K. Arrowsmith, M. Barenco, R. J. Mondragon, and M. Woolf, "The statistics of intermittency maps and dynamical modeling of networks," presented at the 6th Int. Symp. on Mathematical Theory of Networks and Systems, Leuven, Belgium, 2004.
[19] G. Millán, H. Kaschel, and G. lefranc, "Discussion of the analysis of self-similar teletraffic with long-range dependence (LRD) at the network layer level," International Journal of Computer Communications \& Control, vol. V, no. 5, pp. 799-812, Dec. 2010.

[20] G. Millán, E. San Juan, and M. Vargas, "A simplified multifractal model for self-similar traffic flows in high-speed computer networks," Computación y Sistemas, vol. 23, no. 4, pp. 157-1521, 2019.

[21] G. Millán, "Modelado de tráfico basado en procesos multifractales asociativos," Ph.D. dissertation, Departamento de Ingeniería Eléctrica, Universidad de Santiago de Chile, Santiago, Chile, 2013.

[22] G. Millán and G. Lefranc, "A fast multifractal model for self-similar traffic flows in high-speed computer networks," Procedia Computer Science, vol. 17, pp. 420-425, 2013

[23] G. Millán, R. Osorio-Comparán, and G. Lefranc, "Preliminaries on the accurate estimation of the Hurst exponent using time series," arXiv preprint. arXiv: 2103.02091, 2021.

[24] W. Willinger, M. S. Taqqu, R. Sherman, and D. V. Wilson, "Selfsimilarity through high-variability: statistical analysis of Ethernet LAN traffic at the source level," IEEE/ACM Trans. Netw., vol. 5, no. 1, pp. 71-86, Feb. 1997.

[25] H. G. Schuster and W. Just, Deterministic Chaos. An Introduction, 4th Ed., Federal Republic of Germany: Wiley-VHC, 2005, ch. 3, pp. 21-27.

[26] L. G. Samuel, J. M. Pitts, and R. J. Mondragon, "Applications of nonlinear dynamics to network modeling," presented at the 15th UK Teletraffic Symp. Performance Eng. in Inform. Syst., Manchester, 1998.

[27] S. Morita, "Bifurcations in globally coupled chaotic maps," Physics Letters A, vol. 211, no. 5, pp. 258-264, Feb. 1996.

[28] R. Klages, "Comment on "analysis of chaotic motion and its shape dependence in a generalized piecewise linear map"', Physical Review E, vol. 66, no. 1, pp. 018201-1-018201-3, Jul. 2002.

[29] P. Pruthi and A. Erramilli, "Heavy-tailed on/off source behavior and self-similar traffic," in 1995 IEEE Int. Conf. Commun., vol. 1, pp. 445450.

[30] G. Aklilu, J. M. Pitts, and R. J. Mondragon.Modelling the bounds on LRD behavior for IP traffic. Presented at PGNet 2001 [Online]. Available: http://www.cms.livjm.ac.uk/pgnet2001/papers/GAklilu.pdf. 